\title{
New Quantum Limits in Plasmonic Devices
}

\author{
M. Marklund ${ }^{1}$, G. Brodin ${ }^{1}$, L. Stenflo ${ }^{1}$ and C.S. Liu $^{2}$ \\ 1 Department of Physics, Umeå University, SE-901 87 Umeå, Sweden \\ 2 Department of Physics, University of Maryland, College Park, Maryland 20742, USA
}

PACS 73.20.-r - Electron states at surfaces and interfaces

PACS 73.20.Mf - Collective excitations (including excitons, polarons, plasmons and other chargedensity excitations)

\begin{abstract}
Surface plasmon polaritons (SPPs) have recently been recognized as an important future technique for microelectronics. Such SPPs have been studied using classical theory. However, current state-of-the-art experiments are rapidly approaching nanoscales, and quantum effects can then become important. Here we study the properties of quantum SPPs at the interface between an electron quantum plasma and a dielectric material. It is shown that the effect of quantum broadening of the transition layer is most important. In particular, the damping of SPPs does not vanish even in the absence of collisional dissipation, thus posing a fundamental size limit for plasmonic devices. Consequences and applications of our results are pointed out.
\end{abstract}

The excitation and propagation of surface modes at plasma interfaces have long been important in space physics, magnetic confinement fusion, and laboratory plasma physics [1-4]. Moreover, studies of electron oscillation excitations (surface plasmons, surface plasmon polaritons (SPPs) and magnetoplasmons) [5-7] in nanostructured systems [8-11] have recently attracted much interest. It has been found that at condensed matter interfaces, such plasmon excitations could be of crucial importance in future electronic components [13-16], the latter referred to as plasmonic devices $[17,18]$.

Furthermore, the field of quantum plasmas has recently developed rapidly [19]. This field started already in the 1960's, when Pines studied the excitation spectrum of quantum plasmas [20], with a high density and a low temperature as compared to normal plasmas. In such systems, the finite width of the electron wave function makes quantum tunnelling effects crucial, leading to an altered dispersion relation. Since then, a number of theoretical studies of quantum statistical properties of plasmas have been published (see, e.g., Ref. [21] and references therein). For example, Bezzerides \& DuBois presented a kinetic theory for the quantum electrodynamical properties of nonthermal plasmas [22], while Hakim \& Heyvaerts used a covariant Wigner function approach for relativistic quantum plasmas [23]. It has also been shown that, under certain conditions, plasmas can display remarkable properties due to the quantum properties of the constituents. Thus, there are quantum multistream instabilities [24,25], quantum modified Zakharov dynamics $[26,27]$ together with soliton formation and nonlinear quantum interactions [28,29], spin effects on the plasma dispersion [30,31], quantum plasma turbulence [32], ferromagnetic plasma behaviour and Jeans-like instabilities due to quantum effects [33].

Many of the current studies involving quantum plasmas are motivated by the rapid experimental progress and development of new materials, e.g., nanostructured materials [34] and quantum wells [35], and the laboratory realization of ultracold plasmas [36] and experimental demonstration of quantum plasma oscillations in Rydberg systems [37]. Quantum dispersive effects can also be important for diagnostics of inertial fusion plasmas [38]. In parallel, the field of plasmonics and its use of surface waves, such as surface plasmon polaritons (SPPs), has emerged as a new route to electronic devices [12].

In this Letter, methods from quantum plasma physics are used for analyzing SPPs in nanoscale systems. In particular, we determine the dispersion relation for quantum SPPs on a conductor-dielectric interface. It is shown that wave function dispersion introduces an intrinsic damping, even in the absence of collisions. Such damping is due to the irreversible propagation of resonant plasmons towards lower density regions. This is of importance for the short length scales in forthcoming electronic components based on SPPs in waveguide slots. An expression for the damping length, limiting the size of such devices, is presented. For wavelengths of the order of tens of nanometers, the propagation distance for the SPPs is only a fraction of the 
wavelength.

The governing equations for the electrostatic electron dynamics are [19] the continuity equation $\partial_{t} n+\nabla \cdot(n \mathbf{v})=$ 0 , the momentum conservation equation

$$
m n\left(\partial_{t}+\mathbf{v} \cdot \nabla\right) \mathbf{v}=-e n \mathbf{E}-\nabla p-m n \nu \mathbf{v}+\frac{\hbar^{2} n}{2 m} \nabla\left(\frac{\nabla^{2} \sqrt{n}}{\sqrt{n}}\right),
$$

and Poisson's equation $\nabla \cdot\left(\epsilon_{0} \mathbf{E}+\mathbf{P}\right)=n_{i}-n$, where $n$ is the electron density, $n_{i}$ is the ion density (here we treat the lattice constituents in terms of the ion density, in order to demonstrate the analogy to classical plasmas and surface plasmons), $m$ is the electron mass, $\mathbf{v}$ is the electron velocity, $e$ is the magnitude of the electron charge, $\mathbf{E}$ denotes the electric field strength, $p$ is the electron pressure, $\hbar$ is Planck's constant, $\epsilon_{0}$ is the vacuum dielectric constant, $\nu$ is the collisional frequency between the electrons and the ions, $\mathbf{P}=\epsilon_{0}\left(\epsilon_{\mathrm{d}}-1\right) \mathbf{E}$ is the polarization due to bound charges in the system, and $\epsilon_{\mathrm{d}}$ is the permittivity. The last term in Eq. (1), the gradient of the Bohm-de Broglie potential, corresponds to the effect of wave function dispersion.

We consider the ions as stationary, and write the perturbed electric field according to $\delta \mathbf{E}=-\nabla \delta \phi$. Moreover, we assume that the conducting medium is semi-infinite in the positive $x$-direction, so that the stationary background electron density $n_{0}$ is a function of $x$ (see Fig. 1). Next, we linearize the governing equations. In the cold classical case we obtain the SPP dispersion relation $\nabla \cdot[\epsilon(x) \nabla \delta \phi]=0$, where $\epsilon(x)=\epsilon_{\mathrm{d}}(x)-\omega_{\mathrm{p}}^{2}(x) / \omega(\omega+i \nu)$ is the total dielectric function for a cold classical plasma with collision frequency $\nu$, and $\omega_{\mathrm{p}}(x)=\left[e^{2} n_{0}(x) / \epsilon_{0} m\right]^{1 / 2}$ is the electron plasma frequency. We note that the collisional frequency is a function of the ion density. In the general case, we obtain

$$
\partial_{a}\left[\hat{\epsilon}^{a b} \partial_{b} \delta \phi\right]=0
$$

where we use the index notation $\nabla \rightarrow \partial_{a}$ etc., as well as Einstein's summation convention and let all perturbed quantities be of the form $g(x) \exp (i k y-i \omega t)$ [39]. Here the dielectric tensor operator is given by

$\hat{\epsilon}^{a b}=\delta^{a b} \epsilon+\left[\frac{\partial^{a} c_{s}^{2}}{\omega^{2}}-\frac{\omega_{\mathrm{p}}^{2} \partial^{a}}{4 m^{2}}\left\{\frac{\hbar^{2} \partial_{c}}{\omega_{\mathrm{p}}^{2}}\left[\frac{\omega_{\mathrm{p}}^{2} \partial^{c}}{\omega^{2}}\left(\frac{1}{\omega_{\mathrm{p}}^{2}}\right)\right]\right\}\right] \partial^{b} \epsilon_{\mathrm{d}}$,

where $c_{s}=\left[\left.(d p / d n)\right|_{n_{0}} / m\right]^{1 / 2}$ is the sound speed. We will from here on consider a pressure $p=$ $\left(4 \pi^{2} \hbar^{2} / 5 m\right)(3 / 8 \pi)^{2 / 3} n^{5 / 3}$ of a non-relativistic degenerate electron gas, so that the sound speed becomes $c_{s}=$ $(2 \pi \hbar / \sqrt{3} m)\left(3 n_{0} / 8 \pi\right)^{1 / 3}$.

We consider a plasma which can be divided into three regions (see Fig. 1). For $x<0$, the unperturbed plasma density is zero. For $0 \leq x \leq d$, we have a transition layer where the plasma density is a monotonously increasing function of $x$. Lastly, the plasma density is constant for $x>d$. Furthermore, for the surface waves to be only

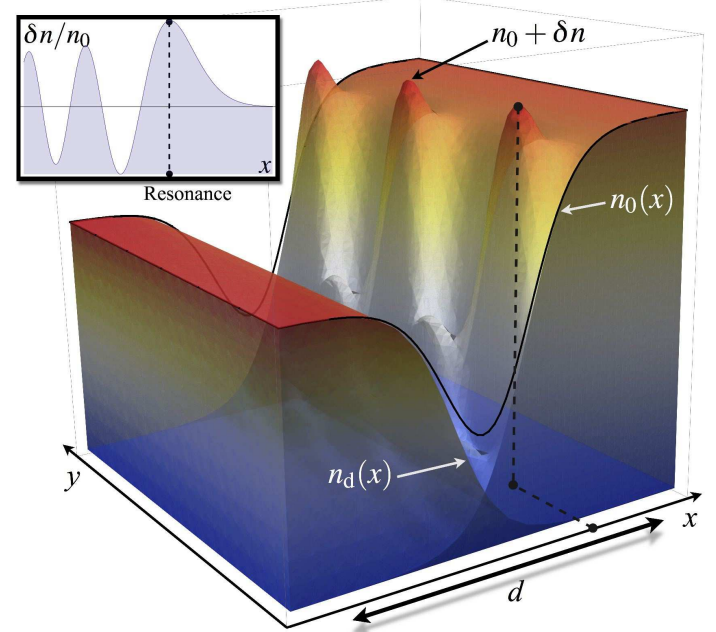

Fig. 1: The structure of the electron density profile for the SPP excitation. The width $d$ of the transition layer is determined by quantum effects. A dielectric material, with electron density $n_{\mathrm{d}}(x)$, is on the left, while the metal with the corresponding plasma oscillations is on the right. The small panel in the upper left corner shows the Airy-like structure of the density perturbations $\delta n / n_{0}$.

weakly damped, the condition $k d \ll 1$ must be fulfilled. The physics inside the transition layer is then essentially one-dimensional, since $k \ll \partial / \partial x$ applies here.

Integrating Eq. (2) across the transition layer, we obtain

$$
\left[\hat{\epsilon}^{x x} \partial_{x} \delta \phi+i k \hat{\epsilon}^{x y} \delta \phi\right]_{0}^{d}=\int_{0}^{d}\left[k^{2} \hat{\epsilon}^{y y} \delta \phi-i k \hat{\epsilon}^{y x} \partial_{x} \delta \phi\right] d x .
$$

Moreover, $\delta \phi(d)$ and $\delta \phi(0)$ are related by

$$
\delta \phi(d)-\delta \phi(0)=\int_{0}^{d} \frac{\partial \delta \phi}{\partial x} d x=-\frac{i e}{\omega} \int_{0}^{d} n_{0}(x) v_{x}(x) d x
$$

The integrals in Eqs. (4) and (5) are correction terms proportional to $k d$. Similarly the off-diagonal quantum terms in the left hand side of (4) are also proportional to one of two small parameters, namely $k^{4} \hbar^{2} / m^{2} \omega_{\mathrm{p}}^{2}$ (from the Bohm-de Broglie potential) or $k^{2} c_{s}^{2} / \omega_{\mathrm{p}}^{2}$ (from the Fermi pressure). If we drop both these quantum terms and let $d \rightarrow 0$, we obtain the wellknown electrostatic dispersion relation $\omega=\omega_{\mathrm{p}}^{(0)}\left(1+\epsilon_{\mathrm{d}}^{(0)}\right)^{-1 / 2}$, where $\epsilon_{\mathrm{d}}^{(0)}=\epsilon_{\mathrm{d}}(x<0)$ is the constant dielectric permittivity for $x<0$, and $\omega_{\mathrm{p}}^{(0)}$ is the plasma freqency at $x=d$. Within this approximation, the electrostatic surface wave has a zero damping as well as a zero group velocity. The quantum terms modify this result, since $d$ now remains finite. To adress this case properly, we must determine $n_{0}(x) v_{x}(x)$ inside the transition layer. Using the approximation $k \ll \partial / \partial x$ inside this region we find

$$
\hat{\epsilon}^{x x}\left[n_{0}(x) v_{x}(x)\right]=-\frac{i \omega k}{e} \hat{\epsilon}^{x x}(0) \delta \phi(0) .
$$

We can here treat $\delta \phi(x)$ as a constant within the layer, in which case the right hand side of (4) reduces to 
$\delta \phi(0) \int_{0}^{d} k^{2} \epsilon(x) d x$. Moreover, when the inhomogeneous derivatives on the left hand side of (44) vanish, the remaining quantum terms become proportional to $\nabla^{2} \delta \phi$. However, for $k^{2} c_{s}^{2} / \omega_{\mathrm{p}}^{2} \ll 1$ and $k^{4} \hbar^{2} / m^{2} \omega_{\mathrm{p}}^{2} \ll 1$, the homogeneous regions are classical to first order, i.e., $\nabla^{2} \delta \phi=0$. The dispersion relation for SPPs then becomes

$$
\omega=\frac{\omega_{\mathrm{p}}^{(0)}}{\left(1+\epsilon_{\mathrm{d}}^{(0)}\right)^{1 / 2}}\left[1+\frac{k}{2} \int_{0}^{d}[\epsilon(x)+q(x)] d x\right]
$$

where $q(x)$ is the solution to Eq. (6) for the plasmon field inside the transition layer, defined by $q(x)=$ $\operatorname{ien}_{0}(x) v_{x}(x) / \omega k \delta \phi(0)$.

The integral contribution in (7) depends on the equilibrium profile. Before turning our attention to the full quantum equilibrium profile we consider the following.

i) Classical case. Here a finite width of the electron distribution in the transitions layer is determined by a finite width of the ion profile. From (6) we obtain $q(x)=\epsilon(0) / \epsilon(x)$. The singularity in this equation can be dealt with using the Landau prescription, which results in an imaginary contribution to the integral, $\operatorname{Im}\left\{\int_{0}^{d} q d x\right\}=i \pi \epsilon(0)(\partial \epsilon / \partial x)_{\epsilon=0}^{-1}$ in Eq. (7), where the subscript indicates that the derivative should be evaluated at the resonant surface where $\epsilon=0$. The imaginary part representing damping of the SPP due to energy transfer to the resonant plasmons in the transition layer.

ii) Fermi pressure effects. Assuming that the width is determined by the ion-density profile we drop the Bohmde Broglie potential. In this case, (6) has the solution $q(x)=\operatorname{Gi}(x d \epsilon / d x)+i \operatorname{Ai}(x d \epsilon / d x)$, where we for simplicity assume that $\epsilon(x)$ is a linear function. Here $\mathrm{Gi}$ and $\mathrm{Ai}$ denote solutions to the inhomogeneous and homogeneous Airy equation [40], respectively.The proper causual solution has been found by taking the nondivergent solution to Eq. (6) that represents resonant plasmons propagating towards lower densities. Due to negligible reflection of the plasmons at the dielectric interface, the energy is irreversibly lost, even in the absence of dissipation. As a consequence, the damping of the surface wavecoincides between cases $i$ and ii.

Next, we investigate the equibrium density profile in the transition layer, determined by

$$
\frac{d}{d \bar{x}}\left\{\epsilon_{\mathrm{d}} \frac{d}{d \bar{x}}\left[\eta \bar{n}^{2 / 3}-\frac{1}{\sqrt{\bar{n}}} \frac{d^{2} \sqrt{\bar{n}}}{d \bar{x}^{2}}\right]\right\}=\bar{n}-H(-x),
$$

where we have introduced the normalized distance $\bar{x}=x /\left(\hbar / \sqrt{2} m \omega_{\mathrm{p}}^{(0)}\right)^{1 / 2}$, the normalized density $\bar{n}=$ $n_{0}(x) / n_{0}^{(0)}$, taken the normalized ion-density as a step function $H(-\bar{x})$, and introduced the dimensionless parameter $\eta \equiv 4 \pi^{2}\left(\hbar / \sqrt{2} m \omega_{\mathrm{p}}^{(0)}\right)\left(3 n_{0}^{(0)} / 8 \pi\right)^{2 / 3}$. The relative importance of the the Fermi pressure and the Bohm-de Broglie potential is determined by the parameter $\eta$. We first consider the case $\eta \ll 1$, i.e., the Bohm-de Broglie potential dominates over the Fermi pressure. Noting that $\bar{n}-H(-\bar{x})$ is of order unity after passing the ion density step, the normalized width $\bar{d}$ must be of order unity, i.e., in dimensional units we have $d \sim\left(\hbar / m \omega_{\mathrm{p}}^{(0)}\right)^{1 / 2}$. On the other hand, assuming a dominating Fermi pressure, i.e., $\eta \gg 1$, the width becomes $d \sim\left(\eta \hbar / m \omega_{\mathrm{p}}^{(0)}\right)^{1 / 2}$. For a solid state plasma, which is the case of most interest, $\eta$ is of order unity, and thus we get $d \sim\left(\hbar / m \omega_{\mathrm{p}}^{(0)}\right)$ independently of which of the two estimates we use. Assuming $\eta \sim 1$, we can deduce a number of properties of the resonant plasmon field inside the transition layer using the solutions to (6).

With $n_{0}(x)$ determined by (8), we can now treat the full quantum case. We start with the simple observation that $q(x)$ is independent of $k$, as terms involving $k$ can be neglected in (6) due to $k \ll \partial / \partial x$. Solving Eq. (6) to find a full solution for $q(x)$ is a nontrivial task, requiring a solution to the equilibrium equation (8), that has to be found numerically. However, a qualitative understanding can be obtained by Taylor expanding Eq. (6) close to the classical plasmon resonance $\epsilon(x)=0$. We obtain an Airy like behavior of the plasma oscillations along the $x$ direction in the transition layer, depicted in the upper left panel of Fig. 1. The full quantum case gives two major changes to the classical and Fermi pressure cases. Firstly, the inhomogeneities in the transition layer shifts the plasmon resonance somewhat towards higher densities. Secondly, the typical plasmon wavelength in the $x$-direction becomes comparable to the width of the transition layer. Using this as a basis for an estimate, together with the condition that no plasmons propagate towards higher densities in the transition layer, we find that $\int_{0}^{d} q(x) d x \approx$ $(\pi \hbar / m \omega)^{1 / 2}(0.6+2 i)$. The uncertainty in this expression is directly linked to the uncertainty in the unperturbed density profile and the expression for the transition layer width $d$. Making a similar estimate of the term $\int_{0}^{d} \epsilon(x) d x$ in (77), we use a linear profile for $\epsilon(x)$, in which case we obtain $\int_{0}^{d} \epsilon(x) d x=(\pi \hbar / m \omega)^{1 / 2}[\epsilon(x=d)-\epsilon(x=0)] \approx 0$, where the last equality follows from the dispersion relation (77). A more accurate density profile gives a finite contribution. However, the plasmon coupling term $\propto \int_{0}^{d} q(x) d x$ will still dominate. Thus, we can write our final dispersion relation for the SPPs as

$$
\omega \approx \frac{\omega_{\mathrm{p}}^{(0)}}{\left(1+\epsilon_{\mathrm{d}}^{(0)}\right)^{1 / 2}}\left[1+(0.6+2 i)\left(\frac{\hbar k^{2}}{m \omega_{\mathrm{p}}^{(0)}}\right)^{1 / 2}\right] .
$$

The group velocity and the damping rate of the electrostatic SPPs are $V_{g}=\partial \omega / \partial k=0.6 V_{q}$ and $\operatorname{Im} \omega=2 k V_{q}$, respectively, where we have introduced the characteristic velocity $V_{q}=\left[\hbar \omega_{\mathrm{p}}^{(0)} / m\left(1+\epsilon_{\mathrm{d}}^{(0)}\right)\right]^{1 / 2}$. In Fig. 2 we display the real part of the dispersion relation (9). Apart from the shortest wavelengths, the dispersive properties are dominated by electromagnetic effects. In the short wavelength limit the group velocity of the classical dispersion relation approaches zero, whereas the quantum corrected value $V_{g}$ approaches a small but nonzero constant value $V_{q}$. Let 


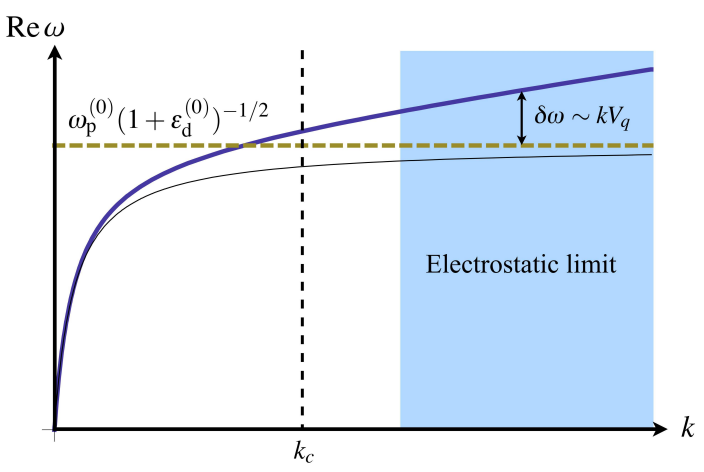

Fig. 2: The classical dispersion relation (thin curve), and the quantum dispersion relation (thick curve) for the SPPs. The dashed horizontal line gives the classical resonance frequency. For wavenumbers larger than $k_{c} \equiv\left(\epsilon_{\mathrm{d}}^{(0)} \omega_{\mathrm{p}}^{(0)}\right)^{5 / 6}\left(m / \hbar c^{4}\right)^{1 / 6}$, the quantum contribution to the group velocity is larger than the classical electromagnetic contribution. We note that quantum damping, as represented by $\operatorname{Im} \omega$ in (9), can be important for wavenumber lower than $k_{c}$.

us point out that there might be classical effects not included in our model that can modify the given picture, e.g. thermal effects which can induce a $k$-dependence of $\epsilon$. We note, however, that within a fluid model, the density perturbation of the surface wave approaches zero, which leads to negligible modifications of our model. Within a kinetic picture, on the other hand, thermal effects induce classical modifications with a $k$-dependence of $\epsilon$, which might lead to modifications of the dispersive properties also in the short wavelength limit.

However, the main importance of the dispersion relation (9) concerns the dissipative (i.e. imaginary) part, which can have significant consequences for the rapidly emerging fields of plasmonics $[15,17,18]$, where plasmon wave propagation along metallic-dielectric interfaces is studied as a means to pave the way for even smaller and faster electronc circuits $[15,16]$. Naturally, energy losses must be minimized if this undertaking is to be successful. Currently the propagation distances of the SPPs range from a few 100 nanometers up to tens of microns [15]. The way to improve performance has so far been to consider wavguide slots $[15,17,18]$, where most of the energy is distributed in a dielectric, limiting the collisional losses in the metal surface. By cooling the system collisional losses can be further minimized [41]. However, when reducing the size towards the nanoscale, the wavelength of the SPP is also decreased. Assuming that collisional effects are minimized when approaching such a regime, our treatment of quantum losses becomes important. For such nano-sized system, a damping of the order $\operatorname{Im}(\omega) /|\omega| \sim k\left(\hbar / m \omega_{\mathrm{p}}\right)^{1 / 2}$, as given by Eq. (9), becomes crucial, and sets a fundamental limiting factor for how small systems that can be designed. The damping length of the SPP due to quantum effects is given by $\delta_{\mathrm{SP}}=V_{g, \mathrm{em}} / \operatorname{Im} \omega$, where $V_{g, \mathrm{em}} \approx\left(\epsilon_{\mathrm{d}}^{(0)} / c\right)^{2}\left(\omega_{\mathrm{p}}^{(0)} / k\right)^{3}$ is the group velocity including electromagnetic effects [4] in the short wavelength region, while $\operatorname{Im} \omega$ is given by (9).
It should be stressed that the electromagnetic contribution to the group velocity dominates over the quantum induced contribution $V_{q}$ for wavelengths $\lambda \geq 30 \mathrm{~nm}$. Assuming that the dielectric consists of $\mathrm{SiO}_{2}$, we have [42] $\epsilon_{\mathrm{d}}^{(0)} \sim 3-5$, and with the plasma frequency of the metal as $\omega_{\mathrm{p}}^{(0)} \approx 4 \times 10^{15} \mathrm{~s}^{-1}$, we obtain

$$
\delta_{\mathrm{SP}} \approx\left(\frac{\lambda}{100 \mathrm{~nm}}\right)^{4} \mu \mathrm{m}
$$

where $\lambda=2 \pi / k$ is the wavelength. Thus, due to the strong wavelength dependence in (10), $\mu \mathrm{m}$-waves can propagate without significant quantum damping, while decreasing the scale much below the $\mu \mathrm{m}$ regime will affect the effective propagation distance. For example, for $\lambda \sim 30 \mathrm{~nm}$ the damping length $\delta_{\mathrm{SP}} \sim 10 \mathrm{~nm}$. Although different geometries may affect the possibilities to design smaller devices [15], our result (10) is robust, and its consequences must therefore be considered in the design of plasmonic devices.

\section{REFERENCES}

[1] O. M. Gradov and L. Stenflo, Phys. Rep. 94, 111 (1983).

[2] M. Y. Yu, Phys. Rev. A 28, 1855 (1983).

[3] L. Stenflo, Phys. Scripta T63, 59 (1996).

[4] Yu. M. Aliev and G. Brodin, Phys. Rev. A 42, 2374 (1990).

[5] P. J. Feibelman, Phys. Rev. Lett. 30, 975 (1973).

[6] P. J. Feibelman, Phys. Rev. B 9, 5077 (1974).

[7] K.-D. Tsuei, E. W. Plummer, and P. J. Feibelman, Phys. Rev. Lett. 63, 2256 (1989).

[8] S. Holland, Ch. Heyn, D. Heitmann, et al., Phys. Rev. Lett. 93, 186804 (2004).

[9] G. Sukhodub, F. Hohls, and R. J. Haug, Phys. Rev. Lett. 93, 196801 (2004).

[10] K. Kneipp, Physics Today 60, Issue 11, 40 (2007).

[11] D. E. Chang, A. S. Sørensen, E. A. Demler, et al., Nature Phys. 3, 807 (2007).

[12] H. A. Atwater, Sci. Am. 296, 56 (2007).

[13] H. J. Lezec, A. Degiron, E. Devaux, et al., Science 297, 820 (2002).

[14] W. L. Barnes, A. Dereux, and T. W. Ebbesen, Nature 424, 824 (2003).

[15] S. A. Maier and H. A. Atwater, J. Appl. Phys. 98, 011101 (2005).

[16] S. A. Maier, Plasmonics (Springer, New York, 2007).

[17] E. Ozbay, Science 311, 189 (2006).

[18] J. M. Pitarke, V. M. Silkin, E. V. Chulkov, et al., Rep. Prog. Phys. 70, 1 (2007).

[19] G. Manfredi, Fields Inst. Comm. 46, 263 (2005).

[20] D. Pines, J. Nucl. Energy C: Plasma Phys. 2, 5 (1961).

[21] D. Kremp, M. Schlanges, and W.-D. Kraeft, Quantum Statistics of Nonideal Plasmas (Springer, 2005).

[22] B. Bezzerides and D. F. DuBois, Ann. Phys. (N.Y.) 70, 10 (1972).

[23] R. Hakim and J. Heyvaerts, Phys. Rev. A 18, 1250 (1978).

[24] F. Haas, G. Manfredi, and M. Feix, Phys. Rev. E 62, 2763 (2000). 
[25] D. Anderson, B. Hall, M. Lisak, et al., Phys. Rev. E 65, 046417 (2002).

[26] L. G. Garcia, F. Haas, L. P. L. de Oliviera, et al., Phys. Plasmas 12, 012302 (2005).

[27] M. Marklund, Phys. Plasmas 12, 082110 (2005).

[28] P. K. Shukla and B. Eliasson, Phys. Rev. Lett. 96, 245001 (2006).

[29] P. K. Shukla and B. Eliasson, Phys. Rev. Lett. 99, 096401 (2007).

[30] M. Marklund and G. Brodin, Phys. Rev. Lett. 98, 025001 (2007).

[31] G. Brodin and M. Marklund, New J. Phys. 9, 277 (2007).

[32] D. Shaikh and P. K. Shukla, Phys. Rev. Lett. 99, 125002 (2007).

[33] G. Brodin and M. Marklund, Phys. Rev. E 76, 055403(R) (2007).

[34] H. G. Craighead, Science 290, 1532 (2000).

[35] G. Manfredi and P.-A. Hervieux, Appl. Phys. Lett. 91, 061108 (2007).

[36] M. P. Robinson, B. Laburthe Tolra, M. W. Noel, et al., Phys. Rev. Lett. 85, 4466 (2000).

[37] R. S. Fletcher, X. L. Zhang, and S. L. Rolston, Phys. Rev. Lett. 96, 105003 (2006).

[38] S. H. Glenzer, O. L. Landen, P. Neumayer, et al., Phys. Rev. Lett. 98, 065002 (2007).

[39] P. K. Kaw and J. B McBride, Phys. Fluids 13, 1784 (1970).

[40] M. Abramowitz and I. A. Stegun, Handbook of Mathematical Functions, (Dover, New York, 1964).

[41] H. Raether, Surface Plasmons (Springer, Berlin, 1988).

[42] E. Palik (ed.), Handbook of Optical Constants in Solids (Academic Press, Inc., New York, 1985). 